\title{
STABILITY OF NONLINEAR OSCILLATIONS \\ OF A SPHERICAL LAYER OF AN IDEAL FLUID
}

\section{K. Andreev}

UDC 532.5:529.5

\begin{abstract}
The nonstationary motion of a spherical layer of an ideal fluid is investigated taking into account the adiabatic distribution of gas pressure in the internal cavity. The existence of nonlinear oscillations of the layer is established, and their period is determined. It is shown that there is only one equilibrium state of the layer. Amplitude equations taking into account the action of capillary forces on the surfaces of the layer in a linear approximation are obtained and used to study the stability of nonlinear oscillations of the layer. The limiting cases of a spherical bubble and soap film are considered.
\end{abstract}

Keywords: ideal fluid, interface, nonlinear oscillations, small perturbations, stability.

DOI: $10.1134 /$ S0021894419020111

\section{MAIN MOTION AND ITS ANALYSIS} formulas [1]

The radial motion of an ideal incompressible fluid in spherical coordinates $(r, \theta, \varphi)$ is described by the

$$
u=\frac{\Phi(t)}{r^{2}}, \quad \frac{p}{\rho}=\frac{\Phi^{\prime}(t)}{r}-\frac{\Phi^{2}(t)}{2 r^{4}}+f(t),
$$

where $\rho>0$ is the density of the liquid and $\Phi(t)$ and $f(t)$ are arbitrary functions of time; the prime denotes differentiation with respect to $t$.

Solution (1.1) is used to describe the dynamics of a spherically symmetric layer of an ideal fluid with free boundaries. Suppose that $r_{1}(t)$ and $r_{2}(t)\left[r_{1}(0)=r_{10}\right.$ and $r_{2}(0)=r_{20}$, where $\left.r_{10}<r_{20}\right]$ are the free inner and outer boundaries of the layer, $p_{\infty}(t)$ is the pressure outside the liquid layer, and $p_{1}(t)$ is the pressure in the gas cavity $0 \leqslant r \leqslant r_{1}(t)$. From the dynamic condition on the free boundaries of the layer, we obtain

$$
\Phi^{\prime}\left(\frac{1}{r_{1}}-\frac{1}{r_{2}}\right)+\frac{\Phi^{2}}{2}\left(\frac{1}{r_{2}^{4}}-\frac{1}{r_{1}^{4}}\right)+\frac{2}{\rho}\left(\frac{\sigma_{1}}{r_{1}}+\frac{\sigma_{2}}{r_{2}}\right)=\frac{1}{\rho}\left[p_{1}(t)-p_{\infty}(t)\right]
$$

where $\sigma_{1} \geqslant 0$ and $\sigma_{2} \geqslant 0$ are constant surface tension coefficients.

From the kinematic conditions on the free boundaries of the layer

$$
\frac{d r_{1}}{d t}=\frac{\Phi(t)}{r_{1}^{2}}, \quad \frac{d r_{2}}{d t}=\frac{\Phi(t)}{r_{2}^{2}},
$$

we obtain the integral law of conservation of the volume of the layer:

$$
r_{2}^{3}(t)-r_{1}^{3}(t)=r_{20}^{3}-r_{10}^{3} .
$$

Institute of Computational Modeling, Siberian Branch, Russian Academy of Sciences, Krasnoyarsk, 660036 Russia. Siberian Federal University, Krasnoyarsk, 660036 Russia; andr@icm.krasn.ru. Translated from Prikladnaya Mekhanika i Tekhnicheskaya Fizika, 2019, Vol. 60, No. 2, pp. 137-147, March-April, 2019. Original article submitted October 2, 2018; revision submitted October 2, 2018; accepted for publication October 29, 2018. 
If $p_{1}(t)$ and $p_{\infty}(t)$ are specified, $\Phi(0)=\Phi_{0}, r_{1}(0)=r_{10}, r_{2}(0)=r_{20}$, and the motion of the spherical layer is completely determined by solving the Cauchy problem for system (1.2), (1.3). However, the order of this system can be reduced by using integral (1.4).

Approximate expressions for $r_{1,2}(t)$ and $\Phi(t)$ were derived by Hunt [2] for $p_{1}(t)=0, p_{\infty}(t)=0$, and $\sigma_{1}=\sigma_{2}=0$ in the case of a thin layer, where $\left(r_{20}^{3}-r_{10}^{3}\right) / r_{10}^{3} \ll 1$, and in the case of a thick layer, where the opposite inequality holds. In addition, he performed [2] an analysis of the stability of this motion. An example of constructing an exact solution of the equations of hydrodynamics using the Lagrangian coordinate method in the case of $\sigma_{1}=\sigma_{2}=0$ is given in [3]. The collapse of a spherical layer under the action of only capillary forces for $\sigma_{1}=\sigma_{2} \neq 0, p_{1}(t)=0, p_{\infty}(t)=0$ was studied by Klassen [4]. The evolution of a spherical layer and small perturbations of its free boundaries at $\sigma_{1} \neq 0, \sigma_{2} \neq 0, p_{1}(t)=0, p_{\infty}(t)=0$ was studied by Andreev [5]. A review of studies in which solution (1.1) is used to study various problems of motion and the stability of spherical layers and bubbles is given in [6].

We assume that the pressure in the cavity is distributed adiabatically

$$
p_{1}=p_{0}\left(V_{0} / V(t)\right)^{\gamma},
$$

where $p_{0}=$ const $>0, \gamma>1$ is the adiabatic exponent, and $V_{0}=4 \pi r_{10}^{3} / 3$ and $V(t)=4 \pi r_{1}^{3}(t) / 3$ are the initial and current volumes of the cavity.

We introduce the dimensionless variables $y(\tau)=r_{1} / r_{10}$ and $\tau=t / t_{0}$ ( $t_{0}$ is characteristic time) and the parameters (hereinafter, $p_{\infty}=$ const $\geqslant 0$ )

$$
\varepsilon=\left(\frac{r_{20}}{r_{10}}\right)^{3}-1, \quad \mathrm{We}_{j}=\frac{\sigma_{j} t_{0}^{2}}{\rho r_{10}^{3}}, \quad a=\frac{p_{0} t_{0}^{2}}{\rho r_{10}^{2}}, \quad b=\frac{p_{\infty} t_{0}^{2}}{\rho r_{10}^{2}} .
$$

The parameters $\varepsilon>0$ and $a \geqslant 0$ characterize the initial thickness of the layer and the initial internal pressure, the parameter $b \geqslant 0$ is the external pressure; $\mathrm{We}_{j} \geqslant 0$ are the Weber numbers.

Eliminating the function $\Phi(t)$ from the first equality (1.3) and Eq. (1.2), we obtain a second-order nonlinear equation for the dimensionless inner radius of the layer $y$ :

$$
y\left(1-\frac{y}{\left(\varepsilon+y^{3}\right)^{1 / 3}}\right) y^{\prime \prime}+\left(\frac{3}{2}-\frac{2 y}{\left(\varepsilon+y^{3}\right)^{1 / 3}}+\frac{y^{4}}{2\left(\varepsilon+y^{3}\right)^{4 / 3}}\right) y^{\prime 2}+\frac{2 \mathrm{We}_{1}}{y}+\frac{2 \mathrm{We}_{2}}{\left(\varepsilon+y^{3}\right)^{1 / 3}}-\frac{a}{y^{3 \gamma}}+b=0
$$

with the initial data

$$
y(0)=1, \quad y^{\prime}(0)=y_{0} \equiv \Phi_{0} t_{0} / r_{10}^{3} .
$$

Thus, the motion of the spherical layer is determined by six dimensionless parameters: $\varepsilon, \mathrm{We}_{1}, \mathrm{We}_{2}, a, b$, and $y_{0}$. Multiplying Eq. (1.6) by $2 y^{2} y^{\prime}$, we determine its first integral (energy conservation law):

$$
\begin{gathered}
y^{\prime 2}=\frac{C-f(y)}{y^{3}\left(1-y /\left(\varepsilon+y^{3}\right)^{1 / 3}\right)} ; \\
f(y)=\frac{2}{3}\left(b y^{3}+\frac{a}{\gamma-1} y^{3(1-\gamma)}\right)+2 \mathrm{We}_{1} y^{2}+2 \mathrm{We}_{2}\left(\varepsilon+y^{3}\right)^{2 / 3} .
\end{gathered}
$$

The constant $C$ depends on the initial data (1.7):

$$
C=\left(1-\frac{1}{(\varepsilon+1)^{1 / 3}}\right) y_{0}^{2}+2 \mathrm{We}_{1}+2 \mathrm{We}_{2}(\varepsilon+1)^{2 / 3}+\frac{2}{3}\left(\frac{a}{\gamma-1}+b\right)>0 .
$$

Note that the solution is bounded, e.g., $y \leqslant \sqrt{C /\left(2 \mathrm{We}_{1}\right)}$ for $\sigma_{1} \neq 0$. It is obvious that $f(y) \rightarrow+\infty$ for $y \rightarrow 0$ as $y \rightarrow \infty$ for $f^{\prime \prime}(y)>0$, i.e., the function $f(y)$ from (1.9) is convex downward. The values of $y$ for which $0<f(y)<C$ have a physical meaning. Therefore, there exist $y_{1}>0$ and $y_{2}>0$ such that $f\left(y_{1}\right)=f\left(y_{2}\right)=C$, where $y_{1}<y<y_{2}$, the fluid layer oscillates periodically, and the inner radius $r_{1}=r_{10} y$ varies between two boundary values $r_{1 \text { min }}=$ $r_{10} y_{1} \leqslant r_{1} \leqslant r_{1 \max }=r_{10} y_{2}$. The outer radius varies as follows: $r_{2 \min }=r_{10}\left(y_{1}^{3}+\varepsilon\right)^{1 / 3} \leqslant r_{2} \leqslant r_{2 \max }=r_{10}\left(y_{2}^{3}+\varepsilon\right)^{1 / 3}$. The dimensionless period of self-oscillations equal to

$$
\tau_{*}=\int_{y_{1}}^{y_{2}} \frac{y^{3}\left(1-y\left(\varepsilon+y^{3}\right)^{-1 / 3}\right)}{C-f(y)} d y,
$$

and the dimensional period equals $T=t_{0} \tau_{*}$. 
Remark 1. For $\varepsilon \rightarrow \infty\left(r_{20} \rightarrow \infty\right)$ Eq. (1.6) reduces to the equation of oscillations of a spherical bubble [1]:

$$
y y^{\prime \prime}+\frac{3}{2} y^{\prime 2}+\frac{2 \mathrm{We}_{1}}{y}-\frac{a}{y^{3 \gamma}}+b=0 .
$$

The period of self-oscillations of the bubble is given by the expression

$$
\tau_{*}=\int_{y_{1}}^{y_{2}} \frac{y^{3}}{C_{1}-f_{1}(y)} d y,
$$

where

$$
\begin{gathered}
f_{1}(y)=\frac{2}{3}\left(\frac{a}{(\gamma-1) y^{3(\gamma-1)}}+b y^{3}\right)+2 \mathrm{We}_{2} y^{2}, \\
C_{1}=y_{0}^{2}+2 \mathrm{We}_{2}+\frac{2}{3}\left(\frac{a}{\gamma-1}+b\right), \quad f_{1}\left(y_{1,2}\right)=C_{1} .
\end{gathered}
$$
obtain

In the other limiting case, $r_{20}-r_{10}=h \ll 1$ (soap bubble, film), assuming that $\sigma_{1}=\sigma_{2}$, from (1.6), we

$$
y^{\prime \prime}+4 \tilde{\mathrm{W}} \mathrm{e}_{1} y-\frac{\tilde{a}}{y^{3 \gamma-2}}+\tilde{b} y^{2}=0
$$

The period of self-oscillations of the film is

$$
\tau_{*}=\int_{y_{1}}^{y_{2}} \frac{d y}{C_{2}-f_{2}(y)}
$$

where

$$
\begin{gathered}
f_{2}(y)=\frac{2}{3}\left(\frac{\tilde{a}}{(\gamma-1) y^{3(\gamma-1)}}+\tilde{b} y^{3}\right)+4 \tilde{\mathrm{We}}{ }_{1} y^{2}, \\
C_{2}=y_{0}^{2}+4 \tilde{\mathrm{We}} \mathrm{e}_{1}+\frac{2}{3}\left(\frac{\tilde{a}}{\gamma-1}+\tilde{b}\right), \quad f_{2}\left(y_{1,2}\right)=C_{2} .
\end{gathered}
$$

In (1.11), in the expressions for the parameters $\tilde{W} e_{1}, \tilde{a}$, and $\tilde{b}$, the density $\rho$ from $(1.5)$ is replaced by $\tilde{\rho}=\rho h=$ const for $h \rightarrow 0$, and $r_{10}^{3}$ and $r_{10}^{2}$ are replaced by $r_{10}^{2}$ and $r_{10}$.

\section{EQUILIBRIUM STATE} equation

In the case of the equilibrium state, $y^{\prime}=0$ and $y^{\prime \prime}=0$ and Eq. (1.6) reduces to the nonlinear algebraic

$$
\frac{a_{1}}{y^{3 \gamma}}-\frac{a_{2}}{\left(\varepsilon+y^{3}\right)^{1 / 3}}-\frac{1}{y}=a_{3}
$$

with new dimensionless parameters $a_{1}=p_{0} r_{10} /\left(2 \sigma_{1}\right), a_{2}=\sigma_{2} / \sigma_{1}$, and $a_{3}=p_{\infty} r_{10} /\left(2 \sigma_{1}\right)$. Note that in the case of the absence of the internal pressure $\left(p_{0}=0\right)$, the layer cannot be in equilibrium. It can be proved that Eq. (2.1) has the unique solution $y_{c}<a_{1}^{1 /(3 \gamma-1)}$, where $y_{c} \rightarrow 0$ as $a_{3} \rightarrow \infty$. Note also that Eq (2.1) leads to $y_{c}>\left[a_{1} /\left(1+\delta a_{1}^{1 /(3 \gamma-1)}\right)\right]^{1 /(3 \gamma-1)}$, where $\delta=\varepsilon^{-1 / 3} a_{2}+a_{3}$.

Remark 2. Similar statements apply to the state of equilibrium of a spherical bubble and soap film [Eqs. (1.10) and (1.11)]. 


\section{EQUATIONS FOR SMALL PERTURBATIONS}

Let $(U(r, \theta, \varphi, t), V(r, \theta, \varphi, t), W(r, \theta, \varphi, t))$, and $P(r, \theta, \varphi, t)$ be perturbations of the velocity vector and the pressure of the main motion (1.1). Then, in the layer $r_{1}(t)<r<r_{2}(t)(0 \leqslant \theta \leqslant \pi$ and $0 \leqslant \varphi \leqslant 2 \pi)$, these functions satisfy the linearized Euler equations

$$
\begin{gathered}
U_{t}+u U_{r}+u_{r} U=-\frac{1}{\rho} P_{r}, \quad V_{t}+u V_{r}+\frac{u}{r} V=-\frac{1}{\rho r} P_{\theta}, \\
W_{t}+u W_{r}+\frac{u}{r} W=-\frac{1}{\rho r \sin \theta} P_{\varphi}, \quad U_{r}+\frac{1}{r} V_{\theta}+\frac{1}{r \sin \theta} W_{\varphi}+\frac{2}{r} U+\frac{\cot \theta}{r} V=0 .
\end{gathered}
$$

We represent the perturbations of the boundaries of the layer in the form $\tilde{r}_{1}=r_{1}(t)+R_{1}(\theta, \varphi, t)$ and $\tilde{r}_{2}=r_{2}(t)+R_{2}(\theta, \varphi, t)$. From the dynamic and kinematic conditions on the inner boundary of the layer at $r=$ $r_{1}(t)$, we obtain [6]

$$
\begin{gathered}
P_{1}(t)-P-p_{r} R_{1}=-\sigma_{1}\left[\frac{2 R_{1}}{r_{1}^{2}}+\frac{1}{r_{1}^{2}}\left(R_{1 \theta \theta}+\cot \theta R_{1 \theta}+\frac{1}{\sin ^{2} \theta} R_{1 \varphi \varphi}\right)\right], \\
R_{1 t}=u_{r} R_{1}+U,
\end{gathered}
$$

where $P_{1}(t)$ is the pressure perturbation $p_{1}(t)$ in the cavity. In a linear approximation, we have

$$
P_{1}(t)=-\frac{3 \gamma p_{0} r_{10}^{3 \gamma}}{4 \pi r_{1}^{3 \gamma+1}} \int_{0}^{2 \pi} \int_{0}^{\pi} R_{1}(\theta, \varphi, t) \sin \theta d \theta d \varphi
$$

At the outer boundary of the layer at $r=r_{2}(t)$, the following conditions should be satisfied [6]:

$$
\begin{gathered}
-P-p_{r} R_{2}=\sigma_{2}\left[\frac{2 R_{2}}{r_{2}^{2}}+\frac{1}{r_{2}^{2}}\left(R_{2 \theta \theta}+\cot \theta R_{2 \theta}+\frac{1}{\sin ^{2} \theta} R_{2 \varphi \varphi}\right)\right], \\
R_{2 t}=u_{r} R_{2}+U .
\end{gathered}
$$

Relations (3.2) and (3.4) are the obtained by linearizing the dynamic and kinematic conditions and extending them to the unperturbed boundaries of the layer.

The initial conditions should be supplemented by Eqs. (3.1)-(3.4) for $t=0$ :

$$
\begin{gathered}
(U, V, W)=\left(U_{0}(r, \theta, \varphi), V_{0}(r, \theta, \varphi), W_{0}(r, \theta, \varphi)\right), \\
R_{1}=R_{10}(\theta, \varphi), \quad R_{2}=R_{20}(\theta, \varphi) .
\end{gathered}
$$

Here the functions $U_{0}, V_{0}$, and $W_{0}$ must satisfy the continuity equation [the last equation of system (3.1)].

\section{AMPLITUDE EQUATIONS}

In the problem of the stability of a spherical layer of an ideal fluid, the angular variables $\theta$ and $\varphi$, and radial variables $r$ and $t$ can be separated.

Let $Y_{l m}(\theta, \varphi)$ be spherical functions. For $l \neq 0$, the solution of problem (3.1)-(3.5) is sought in the form

$$
\begin{gathered}
U=\sum_{l=0}^{\infty} \sum_{m=-l}^{l} a_{l m}(r, t) Y_{l m}(\theta, \varphi), \quad V=\sum_{l=0}^{\infty} \sum_{m=-l}^{l}\left(\frac{b_{l m}(r, t)}{\sqrt{l(l+1)}} \frac{\partial Y_{l m}(\theta, \varphi)}{\partial \theta}+\frac{c_{l m}(r, t)}{\sqrt{l(l+1)} \sin \theta} \frac{\partial Y_{l m}(\theta, \varphi)}{\partial \varphi}\right), \\
W=\sum_{l=0}^{\infty} \sum_{m=-l}^{l}\left(\frac{b_{l m}(r, t)}{\sqrt{l(l+1)} \sin \theta} \frac{\partial Y_{l m}(\theta, \varphi)}{\partial \varphi}-\frac{c_{l m}(r, t)}{\sqrt{l(l+1)}} \frac{\partial Y_{l m}(\theta, \varphi)}{\partial \theta}\right), \\
\left(P, R_{1}, R_{2}\right)=\sum_{l=0}^{\infty} \sum_{m=-l}^{l}\left(d_{l m}(r, t), \alpha_{l m}(t), \beta_{l m}(t)\right) Y_{l m}(\theta, \varphi) .
\end{gathered}
$$


Substituting (4.1) into (3.1) and taking into account the properties of spherical functions, we obtain

$$
\begin{array}{rlrl}
\frac{\partial a_{l m}}{\partial t}+\frac{\partial}{\partial r}\left(u a_{l m}\right)+\frac{1}{\rho} \frac{\partial d_{l m}}{\partial r} & =0, & & \frac{\partial b_{l m}}{\partial t}+\frac{u}{r} \frac{\partial}{\partial r}\left(r b_{l m}\right)+\frac{\sqrt{l(l+1)}}{\rho r} d_{l m}=0, \\
\frac{\partial c_{l m}}{\partial t}+\frac{u}{r} \frac{\partial}{\partial r}\left(r c_{l m}\right) & =0, & \frac{1}{r} \frac{\partial}{\partial r}\left(r^{2} a_{l m}\right)-\sqrt{l(l+1)} b_{l m}=0 .
\end{array}
$$

The perturbation of the internal pressure is found by the formula

$$
P_{1}(t)=-\frac{3 \gamma \alpha_{00}(t) p_{0} r_{10}^{3 \gamma} \delta_{0}^{l}}{\left[r_{1}(t)\right]^{3 \gamma+1}},
$$

where $\delta_{0}^{l}$ is the Kronecker symbol. In other words, $P_{1}(t) \neq 0$ only in the case of radial perturbations.

For $r=r_{1}(t)(l \geqslant 1)$, boundary conditions (3.2) have the form

$$
d_{l m}=-\left(p_{r}+\frac{(l-1)(l-2) \sigma_{1}}{r_{1}^{2}}\right) \alpha_{l m}, \quad \frac{\partial \alpha_{l m}}{\partial t}=u_{r} \alpha_{l m}+a_{l m}
$$

On the outer boundary for $r=r_{2}(t)$, from (3.4) we obtain

$$
d_{l m}=\left(-p_{r}+\frac{(l-1)(l-2) \sigma_{2}}{r_{2}^{2}}\right) \beta_{l m}, \quad \frac{\partial \beta_{l m}}{\partial t}=u_{r} \beta_{l m}+a_{l m}
$$

For $t=0$, we have

$$
\begin{gathered}
a_{l m}(r, t)=a_{l m}^{0}(r), \quad b_{l m}(r, t)=b_{l m}^{0}(r), \quad c_{l m}(r, t)=c_{l m}^{0}(r), \\
\alpha_{l m}(0)=\alpha_{l m}^{0}, \quad \beta_{l m}(0)=\beta_{l m}^{0} .
\end{gathered}
$$

The right-hand sides in (4.3) are the coefficients of the Fourier series (4.1) of the initial data (3.5) for the system of spherical functions $Y_{l m}(\theta, \varphi)$, and

$$
\frac{1}{r} \frac{\partial}{\partial r}\left(r^{2} a_{l m}^{0}\right)=\sqrt{l(l+1)} b_{l m}^{0} .
$$

Note that the problem for the functions $c_{l m}(r, t)$ for fixed $l$ and $m$ does not depend on the general problem, and the order of the system of amplitude equations reduces.

\section{TRANSFORMATION OF THE AMPLITUDE EQUATIONS}

For an arbitrary spherical harmonics $(l \geqslant 1$ and $m$ are fixed), we set

$$
A=a_{l m}, \quad B=b_{l m}, \quad D=D_{l m}, \quad N_{1}=\alpha_{l m}, \quad N_{2}=\beta_{l m} .
$$

Eliminating the equations for $c_{l m}$ from system (4.2), we obtain

$$
A_{t}+(u A)_{r}+\frac{1}{r} D_{r}=0, \quad H_{t}+u H_{r}+\frac{\sqrt{l(l+1)}}{\rho} D=0, \quad\left(r^{2} A\right)_{r}-\sqrt{l(l+1)} H=0,
$$

where $H=r B$. System (5.2) can be integrated:

$$
\begin{gathered}
A=l C_{1}(t) r^{l-1}-\frac{(l+1) C_{2}(t)}{r^{l+2}}, \quad B=\frac{H}{r}=\sqrt{l(l+1)}\left(C_{1}(t) r^{l-1}+\frac{C_{2}(t)}{r^{l+2}}\right), \\
D=-\rho\left[C_{1}^{\prime}(t) r^{l}+\frac{C_{2}^{\prime}(t)}{r^{l+1}}+\Phi(t)\left(l C_{1}(t) r^{l-3}-\frac{(l+1) C_{2}(t)}{r^{l+4}}\right)\right] .
\end{gathered}
$$

We introduce dimensionless variables $r_{1}=r_{10} y, t=t_{0} \tau, C_{1}=r_{10}^{2-l} \bar{C}_{1} / t_{0}, C_{2}=r_{10}^{l+3} \bar{C}_{2} / t_{0}, N_{1}=r_{10} \bar{N}_{1}$, $N_{2}=r_{10} \bar{N}_{2}, y=y_{1}, y^{\prime}=y_{2}, \bar{N}_{1}=y_{3}, \bar{N}_{2}=y_{4}, \bar{C}_{1}=y_{5}$, and $\bar{C}_{2}=y_{6}$. Then, from Eqs. (1.8), (4.3)-(4.5), (5.1), and (5.3), we obtain the system of first-order ordinary differential equations 


$$
\begin{gathered}
y_{1}^{\prime}=y_{2}, \quad y_{2}^{\prime}=F_{3}, \quad y_{3}^{\prime}=-\frac{2 y_{2} y_{3}}{y_{1}}+l y_{1}^{l-1} y_{5}-(l+1) y_{1}^{-(l+2)} y_{6}, \\
y_{4}^{\prime}=-\frac{2 y_{1}^{2} y_{2} y_{4}}{\left(\varepsilon+y_{1}^{3}\right)^{2 / 3}}+l\left(\varepsilon+y_{1}^{3}\right)^{(l-1) / 3} y_{5}-(l+1)\left(\varepsilon+y_{1}^{3}\right)^{-(l+2) / 3} y_{6}, \\
y_{5}^{\prime}=\frac{y_{1}^{l+1} F_{1}-\left(\varepsilon+y_{1}^{3}\right)^{(l+1) / 3} F_{2}}{y_{1}^{2 l+1}-\left(\varepsilon+y_{1}^{3}\right)^{(2 l+1) / 3}}, \quad y_{6}^{\prime}=\frac{\left(\varepsilon+y_{1}^{3}\right)^{(l+1) / 3} y_{1}^{l+1}}{y_{1}^{2 l+1}-\left(\varepsilon+y_{1}^{3}\right)^{(2 l+1) / 3}}\left[y_{1}^{l} F_{2}-\left(\varepsilon+y_{1}^{3}\right)^{l / 3} F_{1}\right],
\end{gathered}
$$

where

$$
\begin{gathered}
F_{1}=-l y_{1}^{l-1} y_{2} y_{5}+(l+1) y_{1}^{-(l+2)} y_{2} y_{6}-\left(F_{3}-\frac{(l-1)(l+2) \mathrm{We}_{1}}{y_{1}^{2}}\right) y_{3}, \\
F_{2}=-l\left(\varepsilon+y_{1}^{3}\right)^{(l-3) / 3} y_{1}^{2} y_{2} y_{5}+(l+1)\left(\varepsilon+y_{1}^{3}\right)^{-(l+4) / 3} y_{1}^{2} y_{2} y_{6} \\
-\left(y_{1}^{2}\left(\varepsilon+y_{1}^{3}\right)^{-2 / 3} F_{3}+2 \varepsilon y_{1}\left(\varepsilon+y_{1}^{3}\right)^{-5 / 3} y_{2}^{2}+(l-1)(l+2)\left(\varepsilon+y_{1}^{3}\right)^{-2 / 3} \mathrm{We}_{2}\right) y_{4}, \\
F_{3}=y_{1}^{-1}\left(1-\frac{y_{1}}{\left(\varepsilon+y_{1}^{3}\right)^{1 / 3}}\right)^{-1}\left[a y_{1}^{-3 \gamma}-b-\frac{2 \mathrm{We}_{1}}{y_{1}}-\frac{2 \mathrm{We}_{2}}{\left(\varepsilon+y_{1}^{3}\right)^{1 / 3}}-\left(\frac{3}{2}-\frac{2 y_{1}}{\left(\varepsilon+y_{1}^{3}\right)^{1 / 3}}+\frac{y_{1}^{4}}{2\left(\varepsilon+y_{1}^{3}\right)^{4 / 3}}\right) y_{2}^{2}\right] .
\end{gathered}
$$

For system (5.4), we use the following initial data:

$$
y_{1}(0)=1, \quad y_{2}(0)=0, \quad y_{3}(0)=\delta_{1}, \quad y_{4}(0)=\delta_{2}, \quad y_{5}(0)=0, \quad y_{6}(0)=0
$$

( $\delta_{1}$ and $\delta_{2}$ are the small dimensionless amplitudes of perturbations of the layer surfaces at the initial time). The second, fifth, and sixth conditions in (5.6) imply that the initial velocity of the layer surfaces and the initial velocity of the fluid perturbations are zero.

Results of numerical study of the solution of the Cauchy problem (5.5), (5.6) show that for $l \geqslant 1(\gamma=1.4)$, there are fluctuations in the amplitudes of perturbations of the boundaries of the inner and the outer layers (Fig. 1), and the fluctuation period is different from the main period (Fig. 2). With increasing layer thickness (Fig. 3), fluctuations of the amplitudes of perturbations of the inner (and outer) surfaces cease to be periodic, while the period of nonlinear oscillations of the spherical layer increases (Fig. 4).

A similar situation occurs with an increase in the number of the spherical harmonic $l$. Note also that the change in surface tension only affects the period of oscillation. Thus, the nonradial perturbations of nonlinear oscillations of the boundaries of the spherical layer are limited, and these oscillations are therefore stable in this case.

\section{RADIAL PERTURBATIONS}

In the case of radial perturbations, $l=0$ and all unknowns are independent of the angular variables $\theta$ and $\varphi$. Then, we have

$$
U=\frac{a_{00}(t)}{r^{2}}, \quad V=0, \quad P=\frac{\rho a_{00}^{\prime}}{r}-\frac{\rho a_{00} \Phi}{r^{4}}+g(t),
$$

where $g(t)$ is an arbitrary function. The behavior of the function $W(r, t)$ determined from the equation $(r W)_{t}+$ $u(r W)_{r}=0$ does not affect the amplitudes $\alpha_{00}(t)$ and $\beta_{00}(t)$ of perturbations of the layer boundaries. Since $\Phi(t)=r_{1}^{2} r_{1}^{\prime}=r_{2}^{2} r_{2}^{\prime}$ and $p_{r}\left(r_{1,2}, t\right)=-\rho r_{1,2}^{\prime \prime}$, we obtain the system $N_{1}=\alpha_{00}, N_{2}=\beta_{00}, A=a_{00}$, which can be written as

$$
\begin{gathered}
N_{1}^{\prime}=-\frac{2 r_{1}^{\prime} N_{1}}{r_{1}}+\frac{A}{r_{1}^{2}}, \quad N_{2}^{\prime}=-\frac{2 r_{2}^{\prime} N_{2}}{r_{2}}+\frac{A}{r_{2}^{2}} \\
\left(\frac{1}{r_{1}}-\frac{1}{r_{2}}\right) A^{\prime}=\frac{r_{1}^{\prime}}{r_{1}^{2}}\left(1-\frac{r_{1}^{4}}{r_{2}^{4}}\right) A+\left(\frac{2 \sigma_{1}}{\rho r_{1}^{2}}+r_{1}^{\prime \prime}-\frac{3 \gamma p_{0} r_{10}^{3 \gamma}}{\rho r_{1}^{3 \gamma+1}}\right) N_{1}+\left(\frac{2 \sigma_{2}}{\rho r_{2}^{2}}-r_{2}^{\prime \prime}\right) N_{2} .
\end{gathered}
$$




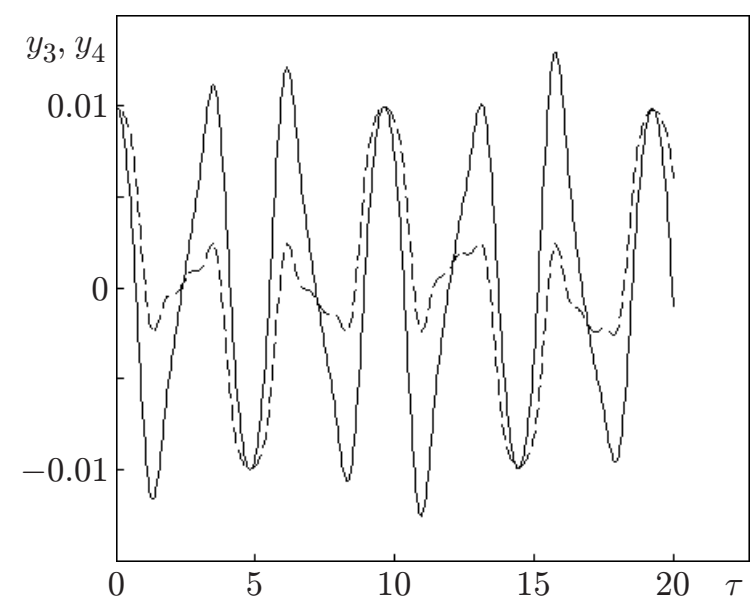

Fig. 1 .

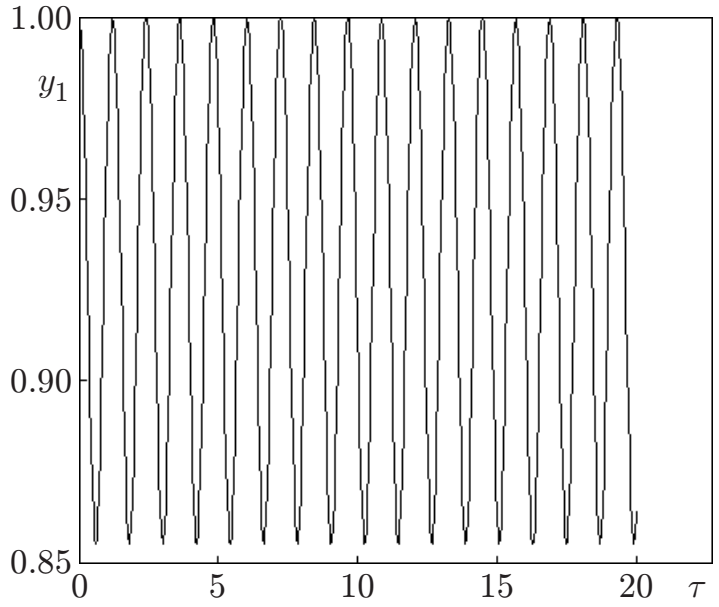

Fig. 2.

Fig. 1. Perturbations of the boundaries of the layer for $l=2, \varepsilon=1, \mathrm{We}_{1}=1.0, \mathrm{We}_{2}=0.1, a=0.1$, $b=0.1$, and $\delta_{1}=\delta_{2}=0.01$ : the solid curve corresponds to the inner boundary, and the dashed curve to the outer boundary.

Fig. 2. Nonlinear perturbations of the inner boundary of the layer for $l=2, \varepsilon=1$, $\mathrm{We}_{1}=1.0$, $\mathrm{We}_{2}=0.1, a=0.1, b=0.1$, and $\delta_{1}=\delta_{2}=0.01$.

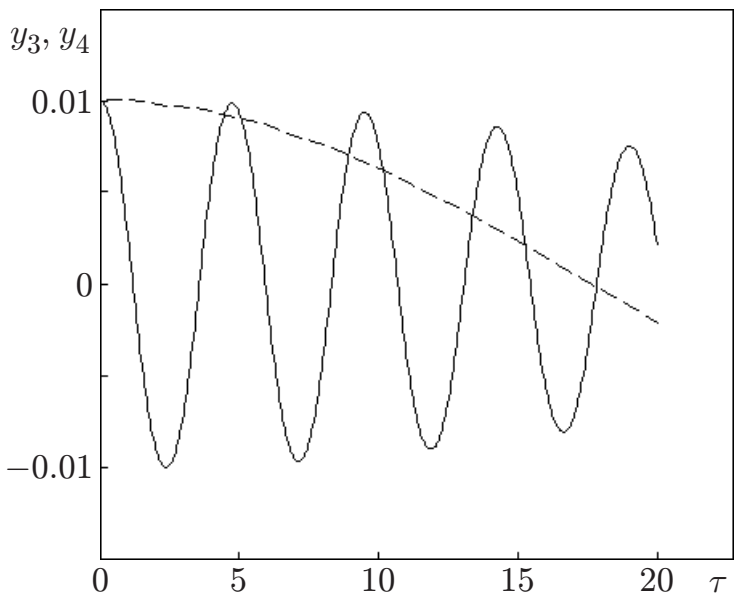

Fig. 3.

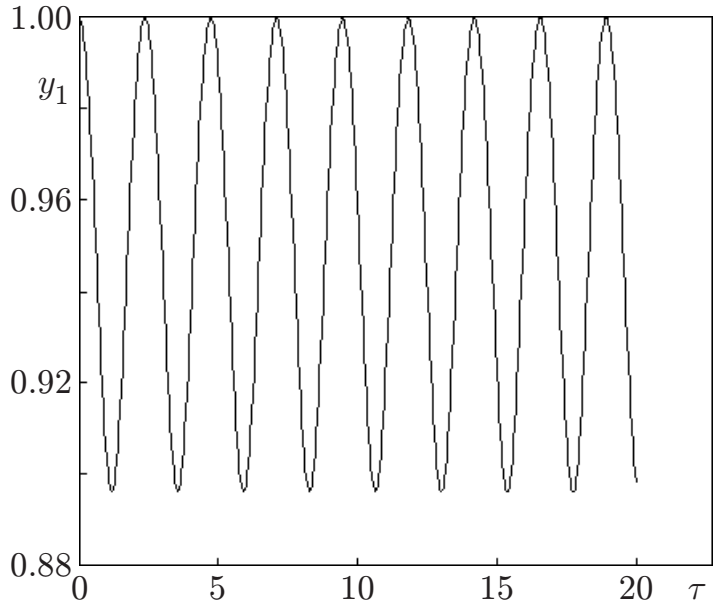

Fig. 4.

Fig. 3. Perturbations of the boundaries of the layer for $l=2, \varepsilon=100, \mathrm{We}_{1}=1.0, \mathrm{We}_{2}=0.1$, $a=0.1, b=0.1$, and $\delta_{1}=\delta_{2}=0.01$ : the solid curve corresponds to the inner boundary, and the dashed curve to the outer boundary.

Fig. 4. Nonlinear perturbations of the inner boundary of the layer for $l=2, \varepsilon=100$, We $_{1}=1.0$, $\mathrm{We}_{2}=0.1, a=0.1, b=0.1$, and $\delta_{1}=\delta_{2}=0.01$.

From the first two equations (6.1) we derive the equalities $A=\left(r_{1}^{2} N_{1}\right)^{\prime}=\left(r_{2}^{2} N_{2}\right)^{\prime}$ or

$$
r_{1}^{2} N_{1}=r_{2}^{2} N_{2} \text {. }
$$

The integration constant must be equal to zero since in the case of radial perturbations, relation (6.2) is a consequence of the law of conservation of volume (1.4). If the perturbation of one of the boundaries is zero at the initial time, e.g., $N_{1}(0)=0$, then $N_{1}(t)=N_{2}(t)=A(t)=0$ for all $t \geqslant 0$ because $A(0)=0$. Using the dimensionless 


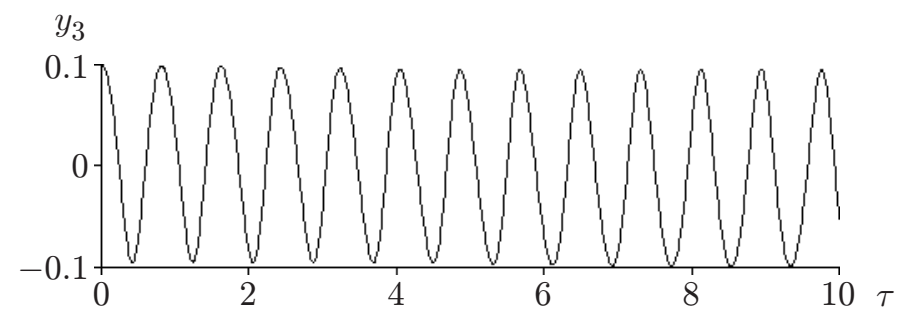

Fig. 5. Perturbations of the layer boundaries for $l=2, \varepsilon=999, \mathrm{We}_{1}=\mathrm{We}_{2}=1$, $a=b=10$, and $\delta_{1}=0.1$.

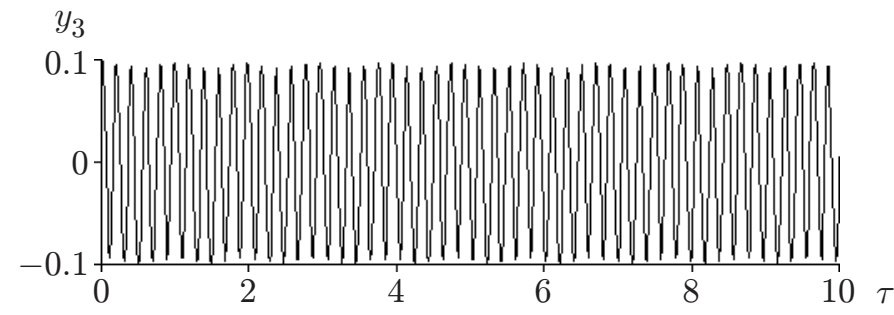

Fig. 6. Perturbations of the inner boundary of the layer for $l=2, \varepsilon=10$, $\mathrm{We}_{1}=$ $\mathrm{We}_{2}=10, a=b=100$, and $\delta_{1}=0.1$.

variables $\tau=t / t_{0}, y_{1}=r_{1} / r_{10}, y_{3}=N_{1} / r_{10}$, and $y_{4}=A t_{0} / r_{10}^{3}$, from (6.1) and (6.2) for $N_{1}(0) \neq 0$, we obtain the system of equations

$$
\begin{gathered}
y_{1}^{\prime}=y_{2}, \quad y_{2}^{\prime}=F_{3}, \quad y_{3}^{\prime}=-\frac{2 y_{2} y_{3}}{y_{1}}+\frac{y_{4}}{y_{1}^{2}}, \\
y_{4}^{\prime}=\frac{y_{2}}{y_{1}}\left(1+\frac{y_{1}}{\left(\varepsilon+y_{1}^{3}\right)^{1 / 3}}+\frac{y_{1}^{2}}{\left(\varepsilon+y_{1}^{3}\right)^{2 / 3}}+\frac{y_{1}^{3}}{\varepsilon+y_{1}^{3}}\right) y_{4} \\
+\frac{y_{1}}{1-y_{1}\left(\varepsilon+y_{1}^{3}\right)^{-1 / 3}}\left[\frac{2 \mathrm{We}_{1}}{y_{1}^{2}}+\frac{2 \mathrm{We}_{2} y_{1}^{2}}{\left(\varepsilon+y_{1}^{3}\right)^{4 / 3}}-\frac{2 \varepsilon y_{1} y_{2}^{2}}{\left(\varepsilon+y_{1}^{3}\right)^{5 / 3}}-\frac{3 \gamma a}{y_{1}^{3 \gamma+1}}+\left(1-\frac{y_{1}^{2}}{\left(\varepsilon+y_{1}^{3}\right)^{2 / 3}}\right) F_{3}\right] y_{3}
\end{gathered}
$$

with the initial conditions

$$
y_{1}(0)=1, \quad y_{2}(0)=0, \quad y_{3}(0)=\delta_{1}, \quad y_{4}(0)=0
$$

[the function $F_{3}$ is taken from (5.5)].

From the solution of the Cauchy problem (6.3), (6.4), it follows that the increase in the amplitudes of perturbations of the layer boundaries are significantly affected by the parameters $a$ and $b$ which characterize the pressure inside and outside the layer. As stated above, the parameter $b$ layer does not have an additional influence on the perturbations of the boundaries. If the parameters $a$ and $b$ have the same values, the perturbations of the boundaries of the layer are oscillatory (Fig. 5). With an increase in $a$ and $b$, the oscillation frequency increases and the perturbations vary in the same range (Fig. 6).

When $a \neq b$, the perturbation amplitudes increase, reaching the maximum value $\max y_{3}=4.435 \cdot 10^{5}$ for $\tau=20$ (Fig. 7). A similar situation occurs when $b>a$, with the layer thickness having not influence on the nature of radial perturbations.

Figure 8 shows the stability region of radial perturbations for $0 \leqslant a \leqslant 25,0 \leqslant b \leqslant 10, l=2, \delta_{1}=0.1$, $\varepsilon=10$, and $\mathrm{We}_{1}=\mathrm{We}_{2}=10$. 


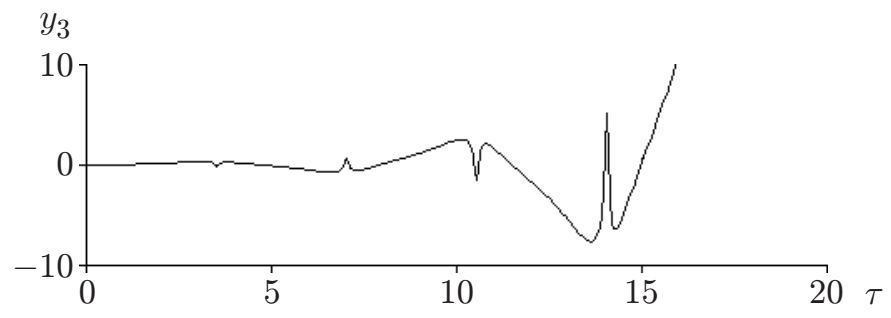

Fig. 7. Perturbations of the inner boundary of the layer for $l=2, \varepsilon=10, \mathrm{We}_{1}=\mathrm{We}_{2}=1$, $a=b=100$, and $\delta_{1}=0.01$.

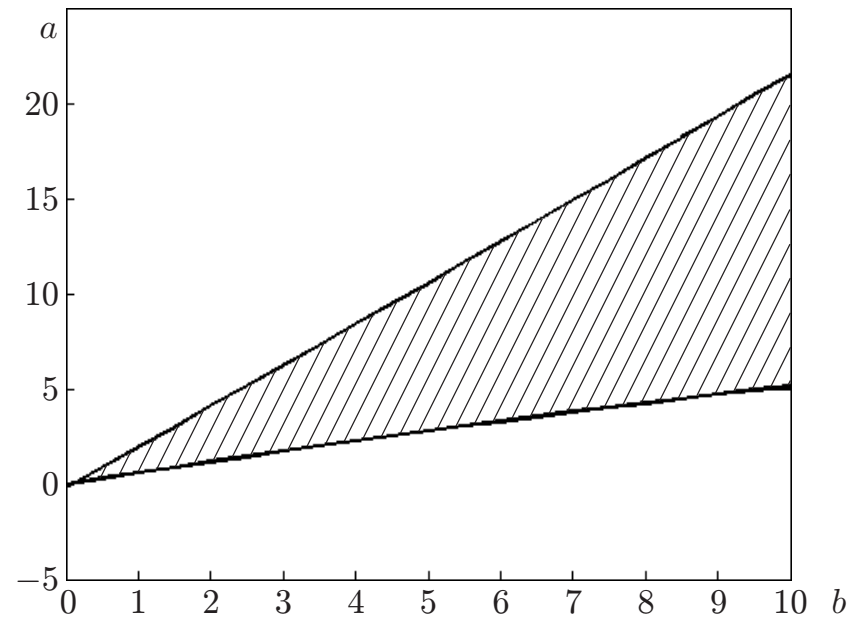

Fig. 8. Stability region of radial perturbations for $0 \leqslant a \leqslant 25$ and $0 \leqslant b \leqslant 10$.

\section{STABILITY OF THE STATIONARY STATE OF THE LAYER}

If the stationary state of the layer is stable, Eq. (2.1) has a unique solution $y_{c}$ :

$$
\frac{2 \mathrm{We}_{1}}{y_{c}}+\frac{2 \mathrm{We}_{2}}{\left(\varepsilon+y_{c}^{3}\right)^{1 / 3}}-\frac{a}{y_{c}^{3 \gamma}}+b=0 .
$$

We first consider radial perturbations. In system (6.3), we must set $y_{1}=y_{c}, y_{2}=0$, and $F_{3}=0$ [the latter follows from equality (7.1)]. Then, system (6.3) reduces to one second-order equation with constant coefficients

$$
y_{3}^{\prime \prime}+\frac{1}{1-y_{1}\left(\varepsilon+y_{c}^{3}\right)^{-1 / 3}}\left(\frac{3 \gamma a}{y_{c}^{3 \gamma+2}}-\frac{2 \mathrm{We}_{1}}{y_{c}^{3}}-\frac{2 \mathrm{We}_{2} y_{c}}{\left(\varepsilon+y_{c}^{3}\right)^{4 / 3}}\right) y_{3}=0 .
$$

Replacing $a / y_{c}^{3 \gamma}$, from (7.1), we obtain the expression in brackets:

$$
\frac{(6 \gamma-2) \mathrm{We}_{1}}{y_{c}^{3}}+\left(\frac{6 \gamma-2}{y_{c}^{2}\left(\varepsilon+y_{c}^{3}\right)^{1 / 3}}+\frac{2 \varepsilon}{y_{c}^{2}\left(\varepsilon+y_{c}^{3}\right)^{4 / 3}}\right) \mathrm{We}_{2}+\frac{3 \gamma b}{y_{c}^{2}} \equiv G\left(y_{c}, \gamma, \varepsilon, \mathrm{We}_{1}, \mathrm{We}_{2}\right)>0 .
$$

Therefore, the equilibrium point $y_{c}$ is stable (center). The oscillation frequency of the layer in the vicinity of this point is found from the formula

$$
\omega_{c}=\left[\left(1-\frac{y_{c}}{\left(\varepsilon+y_{c}^{3}\right)^{1 / 3}}\right)^{-1} G\right]^{1 / 2}
$$

[the function $G$ is taken from (7.2)].

For perturbations of the general form $(l \geqslant 1)$, the characteristic equation is written as

$$
\omega^{4}+A_{1} \omega^{2}+A_{2}=0 .
$$


Here

$$
\begin{gathered}
A_{1}=\frac{(l-1)(l+2)}{y_{c}^{2 l+1}-\left(\varepsilon+y_{c}^{3}\right)^{(2 l+1) / 3}}\left[\frac{1}{y_{c}^{3}}\left(l y_{c}^{2 l+1}+(l+1)\left(\varepsilon+y_{c}^{3}\right)^{(2 l+1) / 3}\right) \mathrm{We}_{1}\right. \\
\left.+\frac{1}{\varepsilon+y_{c}^{3}}\left((l+1) y_{c}^{2 l+1}+l\left(\varepsilon+y_{c}^{3}\right)^{(2 l+1) / 3}\right) \mathrm{We}_{2}\right] \\
A_{2}=\frac{l(l+1)(l-1)^{2}(l+2)^{2} \mathrm{We}_{1} \mathrm{We}_{2}}{y_{c}^{3}\left(\varepsilon+y_{c}^{3}\right)} .
\end{gathered}
$$

Since $A_{1}<0$ and $A_{2}>0$, the squares of the natural frequencies are positive, equal to

$$
\omega_{c}^{2}=\frac{-A_{1} \pm \sqrt{A_{1}^{2}-4 A_{2}}}{2}>0
$$

and depend only on the number $l$, but not on $m$. Each frequency in (7.5) corresponds to $2 l+1$ different natural oscillations. Expression (7.5) vanishes for $l=1$, which corresponds to the translational displacement of the layer as a whole. The lowest possible frequency of the layer is found from expression (7.5), in which the minus sign is used and $l=2$.

Thus, the equilibrium state of the spherical layer is stable.

Remark 3. For $\varepsilon \rightarrow \infty$, from formulas (7.2), (7.3) and (7.4), (7.5), we obtain the following expressions for the oscillation frequency of a spherical bubble in an infinite fluid:

$$
\left(\omega_{c}^{\infty}\right)^{2}=\frac{(6 \gamma-2) \mathrm{We}_{1}}{y_{c}^{3}}+\frac{3 \gamma b}{y_{c}^{2}}, \quad\left(\omega_{c}^{\infty}\right)^{2}=\frac{(l-1)(l+1)(l+2) \mathrm{We}_{1}}{y_{c}^{3}}
$$

$\left[y_{c}\right.$ is the stationary solution of Eq. (1.11)].

Remark 4. If $r_{20}-r_{10}=h \ll 1$, then for $h \rightarrow 0, \sigma_{1}=\sigma_{2}$, and $\tilde{\rho}=\rho h=$ const, Eqs. (7.2) and (7.3) lead to the following expression for the frequency of radial oscillations of a spherical film:

$$
\left(\omega_{c}^{0}\right)^{2}=4(3 \gamma-1) \tilde{W} e_{1}+3 \gamma \tilde{b} y_{c}
$$

[ $y_{c}$ is the stationary solution of Eq. (1.11)].

For nonspherical oscillations, from (7.4) and (7.5), we obtain

$$
\left(\omega_{C}^{0}\right)^{2}=(l-1)(l+2) \tilde{\mathrm{W}} \mathrm{e}_{1} .
$$

\section{CONCLUSIONS}

The nonstationary motion of a spherical layer of an ideal fluid was studied in the case where the pressure in a gas cavity is distributed adiabatically. The motion is described by a second-order strongly nonlinear ordinary differential equation, whose solution depends on six dimensionless parameters. It was proved that under certain conditions for these parameters, the equation has a periodic solution which corresponds to nonlinear oscillations of the layer. The limiting cases of oscillations of a spherical bubble and a soap bubble (film) and the stationary state of the layer were investigated.

The equations of small perturbations of nonstationary motion of the layer with capillary forces on its surfaces were derived. A system of amplitude equations was obtained by special separation of angular variables. Thus system can be integrated over the radial variable, resulting in the Cauchy problem for the system of sixth-order ordinary differential equations for the perturbations. The results of the numerical study of this system show that nonradial perturbations of the layer boundaries are periodic, and their period differs from the oscillation period of the layer for the same values of the physical parameters. If the dimensionless external and internal pressures are not balanced, the radial perturbations of the layer boundaries may increase with time. It was also proved that the equilibrium state of the spherical layer, which is possible only in the presence of the internal pressure, is stable. 


\section{REFERENCES}

1. H. Lamb, Hydrodynamics (Dover, New York, 1932).

2. J. H. Hunt, "Instability in a Spherical Fluid Shell," Appl. Sci. Res. Ser. A 10 (1), 59-77 (1961).

3. L. V. Ovsyannikov, "General Equations and Examples," in The Problem of Unsteady Motion of a Fluid with a Free Boundary (Nauka, Novosibirsk, 1967), pp. 5-75 [in Russian].

4. L. G. Klassen, "On the Collapse of a Cavity in an Ideal Incompressible Fluid by Surface Tension Forces," in Dynamics of Continuous Media, No. 4 (Inst. of Hydrodynamics, Sib. Branch, USSR Acad. of Sci., Novosibirsk, 1973), pp. 106-111.

5. V. K. Andreev, "Small Perturbations of a Spherical Fluid Layer," Prikl. Mekh. Tekh. Fiz., No. 1, 110-117 (1981) [J. Appl. Mech. Tech. Phys. No. 1, 91-97 (1981)].

6. V. K. Andreev, Stability of Unsteady Fluid Motion with a Free Boundary (Nauka, Novosibirsk, 19920 [in Russian]. 